traumatic head injury 4 weeks before. We studied demographic variables, time between beginning of symptoms and medical care, origin in kilometers until hospital, clinical characteristics at admission, including Glasgow and NIHSS. Results. We analyzed 74 men and 101 women with mean age of 65 years. The etiology was hypertension in $77.4 \%$ and localization lobar in 39.4\%. Eighty-five percent receipt medical care after 3 hours and $75.4 \%$ came from a radius $<100 \mathrm{~km}$. Mortality in hospital was $16.6 \%$ with an explicative model of regression that included blood pressure < 130/80 mmHg, intubation, Glasgow < 9 at admission or NIHSS > 15, and hospitalization days. Conclusions. Demographic characteristics, causes, and localization are similar to previously informed series. The time for obtaining medical care is far from ideal, this could delay treatment; allow progression of disease, and then worse prognosis. [REV NEUROL 2008; 46: 67-72]

Key words. Epidemiology. Glasgow. Intracerebral hemorrhage. Mortality. NIHSS.

\title{
Aspectos diferenciales de comorbilidad en pacientes ancianos con demencia tipo Alzheimer o con demencia vascular
}

\author{
F. Formiga ${ }^{\text {a }}$, I. Fort ${ }^{\text {b }}$, M.J. Robles ${ }^{c}$, S. Riu ${ }^{\text {d }}$ D. Rodríguez ${ }^{\mathrm{e}}$, O. Sabartes ${ }^{\mathrm{c}}$
}

ASPECTOS DIFERENCIALES DE COMORBILIDAD EN PACIENTES ANCIANOS

CON DEMENCIA TIPO ALZHEIMER O CON DEMENCIA VASCULAR

\begin{abstract}
Resumen. Introducción. La enfermedad de Alzheimer (EA) y la demencia vascular (DV) son los dos tipos más frecuentes de demencia. En los ancianos con demencia existe una importante comorbilidad asociada que frecuentemente ocasionará una mayor morbimortalidad. Objetivo. Evaluar algunos aspectos de la comorbilidad en pacientes con ambos tipos de demencia para analizar posibles diferencias. Pacientes y métodos. Se evaluaron prospectivamente 365 pacientes mayores de 64 años. De ellos, 289 (79,1\%) tenían EA probable, y 76 DV, probable. Se recogieron variables sociodemográficas, tipo de demencia, indice de Barthel, índice de Lawton, test minimental, número total de medicamentos, antecedentes de hipertensión (HTA), diabetes mellitus (DM), dislipemia, insuficiencia cardiaca, enfermedad pulmonar obstructiva crónica (EPOC) y neoplasia. Resultados. Se trataba de 264 mujeres (72,3\%) y 101 varones. La edad media era de 81,1 \pm 6 años. La media de medicamentos fue de 5,5 $\pm 2,5$. El 54\% recibía tratamiento para la HTA, el 26,6\% para la DM y el 25,8\% para la dislipemia. En el $12,1 \%$ de los pacientes existía el diagnóstico de insuficiencia cardíaca, en el 13,7\% de EPOC y en el 8,2\% coexistía el diagnóstico de neoplasia. En el análisis multivariable se objetivaron algunas diferencias significativas según el tipo de demencia; así, en el grupo de pacientes con DV había predominio de varones, mayor porcentaje de pacientes con más de tres medicamentos y menor porcentaje de ellos con tratamiento específico para su demencia. Conclusiones. Los pacientes ancianos con demencia tienen alta comorbilidad e importante consumo de fármacos de prescripción crónica. Existen algunas variaciones en los pacientes mayores según el tipo de demencia, y destaca un mayor porcentaje de pacientes con polifarmacia en el grupo de demencia vascular. [REV NEUROL 2008; 46: 72-6]
\end{abstract}

Palabras clave. Anciano. Comorbilidad. Demencia vascular. Deterioro cognitivo. Enfermedad de Alzheimer. Polifarmacia.

\section{INTRODUCCIÓN}

La demencia es un síndrome caracterizado por un deterioro adquirido, habitualmente progresivo y persistente de la función cognitiva con repercusión en las actividades de la vida diaria del individuo. Los dos tipos más prevalentes de demencia son, por

Aceptado tras revisión externa: 19.12.07.

${ }^{a}$ UFISS de Geriatría. Servicio de Medicina Interna. Hospital General Universitari de Bellvitge. L'Hospitalet de Llobregat, Barcelona. ${ }^{b}$ Unidad de Atención Integral a la Enfermedad de Alzheimer y otras Demencias. Centre Sociosanitari El Carme. Badalona Serveis Assistencials. Badalona, Barcelona. ${ }^{c}$ Servicio de Geriatría. Instituto de Atención Geriátrica y Sociosanitaria. IMAS. Barcelona. ${ }^{d}$ SRETR. Llars Mundet. Diputació de Barcelona. Barcelona. e Equipo de Evaluación Integral Ambulatoria de Trastornos Cognitivos. Servicio de Geriatría. Consorci Sanitari de Terrassa. Terrassa, Barcelona, España.

Correspondencia: Dr. Francesc Formiga. UFISS de Geriatría. Servicio de Medicina Interna. Hospital General Universitari de Bellvitge. Feixa Llarga, s/n. E-08907 L'Hospitalet de Llobregat (Barcelona). Fax: +34 932607420. E-mail: fformiga@csub.scs.es

(C) 2008, REVISTA DE NEUROLOGÍA orden de frecuencia, la enfermedad de Alzheimer (EA) y la demencia vascular (DV) [1,2].

La asociación entre EA y DV resulta compleja. Ambas incrementan su prevalencia con la edad, y frecuentemente ocurren concomitantemente, existiendo una considerable superposición de síntomas, fisiopatología y comorbilidad entre ambas entidades [3].

En las personas mayores de 65 años, la prevalencia de dos o más enfermedades crónicas como hipertensión, diabetes, artrosis y cardiopatía isquémica es alta. En los ancianos con demencia existe una importante patología asociada [4-10], que frecuentemente ocasionará ingresos hospitalarios y empeoramiento de los síntomas conductuales de la demencia [11]. Además, la comorbilidad asociada a la demencia ha demostrado ser un marcador pronóstico independiente de mortalidad [12]. En un estudio previo realizado en nuestro país, se ha demostrado que los pacientes ancianos con demencia tienen una alta comorbilidad y un importante consumo de fármacos de prescripción crónica [13]. 
Por las importantes posibles repercusiones en la morbimortalidad de los pacientes con demencia, el objetivo del presente estudio es identificar en una población de pacientes ancianos, que cumplan criterios de demencia tipo EA o DV probable, la comorbilidad añadida que presentan para evaluar las similitudes y las diferencias en relación con el tipo de demencia.

\section{PACIENTES Y MÉTODOS}

Se evaluaron prospectivamente 365 pacientes mayores de 64 años, con el diagnóstico de EA o DV probable, evaluados en unidades de atención a pacientes con demencia en servicios de medicina interna o geriatría. No existían criterios de exclusión, excepto la ausencia de un cuidador cualificado con el que pudiera confirmarse la información o que se tratase de una demencia en la que coexistiera EA y afectación vascular cerebral, descrita en ocasiones con el término de demencia mixta.

El estudio se ha descrito ya previamente [13]; se estableció en breve el diagnóstico de demencia siguiendo los criterios del Manual diagnóstico y estadístico de los trastornos mentales, $4 .^{\text {a }}$ edición, (DSM-IV) [14] y para los distintos subtipos de demencias, se utilizaron los criterios del National Institute of Neurological and Communicative Disorders and Stroke-Alzheimer's Disease and Related Disorders Association (NINCDS-ADRDA) en la EA [15] y del National Institute of Neurological Disorders and Stroke y Association Internationale pour la Recherche et l'Enseignement en Neurosciences (NINDS-AIREN) para la DV [16].

Se evaluó el estadio evolutivo de la demencia con la Global Deterioration Scale (GDS), y se complementó con la Functional Assessment Staging (FAST), ambas según Reisberg [17,18]. La GDS realiza una descripción en siete fases (GDS1-GDS7),que se extienden de la normalidad a las fases más graves de la EA. El FAST subdivide las fases GDS6 (cinco subfases) y GDS7 (seis subfases). Las subfases del GDS6 son una disminución en la habilidad para vestirse, bañarse, ir al lavabo, presencia de incontinencia urinaria y presencia de incontinencia fecal; y las del GDS7 corresponden a: reducción del habla a 1-5 palabras por día, pérdida del vocabulario, pérdida de la marcha, incapacidad de estar sentado, incapacidad de sonreír e incapacidad de mantener la cabeza.

Se recogió el número total de fármacos de prescripción crónica. Además, se registró de forma detallada si los enfermos tomaban el tratamiento con fármacos específicos para la demencia (inhibidores de la acetilcolinesterasa, memantina o ambos) en la fecha de inclusión en el estudio. También se analizó si para el control de alguno de los 'síntomas conductuales y psicológicos' asociados a la demencia recibían tratamiento con fármacos neurolépticos (risperidona, quetiapina, olanzapina, haloperidol, levomepromacina u otros). Se incluyó a los pacientes en el grupo en tratamiento con neuroléptico cuando lo hubieran tomado al menos durante 15 días consecutivos y tuviesen el fármaco disponible a demanda, por si fuera preciso para tratar las alteraciones conductuales en caso de presentarse.

Además, en el momento de su inclusión en el estudio, se aplicó un protocolo de recogida de datos, donde se incluía información acerca de variables demográficas, como edad, género, estado civil e identificación del cuidador principal y responsable de la administración del tratamiento. La capacidad funcional para actividades básicas de la vida diaria se midió con el índice de Barthel [19] (IB). El IB es una escala ordinal con una puntuación total de $0-100$. Cortes intermedios del IB nos ayudan a evaluar el grado de dependencia (0-20: total; 21-40: grave; 41-60: moderada; 61-90: leve; y > 90: independiente en domicilio) [20]. Para evaluar la habilidad en actividades instrumentales se utilizó el índice de Lawton-Brody (IL) [21], cuyos valores oscilan entre 0-8 y miden ocho actividades instrumentales de la vida diaria. El estado cognitivo se valoró con el Minimental State Examination (MMSE) [22], cuya puntuación máxima es de 30 (menos de 24 sugiere deterioro cognitivo en mayores de 65 años).

\section{Valoración de la comorbilidad}

Se utilizó el índice de Charlson para evaluar la comorbilidad global (puntúa entre 1 y 6 puntos 19 situaciones clínicas, con una puntuación máxima de gravedad de 37 puntos) [23]. Se investigó sobre la presencia de algunos factores de riesgo cardiovascular, como hipertensión arterial (HTA) -cifras en la visita superiores a $140 / 90 \mathrm{mmHg}$ o tratamiento antihipertensivo actual-, diabetes mellitus (DM) y dislipemia (DL) -ambas si existía el diagnóstico previo y tomaban tratamiento dietético o farmacológico-. Mediante anamnesis al cuidador y registro en la historia clínica se incluyeron los antecedentes de cardiopatía isquémica, insuficiencia cardíaca y enfermedad pulmonar obstructiva crónica (EPOC). De la misma manera, se evaluó también el diagnóstico previo de neoplasia sólida o hematológica.

\section{Estudio estadístico}

Se utilizó el programa informático Excel como base de datos y el SPSS v. 11.0 para el análisis estadístico. Se realizó un análisis descriptivo en que los resultados se expresan con la media y la desviación estándar. La significación estadística de las diferencias se valoró mediante el test $t$ de Student para la comparación de las variables cuantitativas y test no paramétrico en caso de distribución no normal de la variable. La prueba de $\chi^{2}$ con el test exacto de Fisher se utilizó en la comparación de variables cualitativas. Teniendo en cuenta el menor número de pacientes con DV, para una muestra de 76 la potencia del estudio es cercana al $70 \%$ (en concreto $69,71 \%$ ), con una seguridad del $95 \%$, lo que permite obtener diferencias estadísticamente significativas. La existencia de una asociación independiente entre las distintas variables obtenidas en el análisis bivariable se exploró mediante un análisis de regresión logística. Los resultados se consideraron significativos cuando $p<0,05$.

\section{RESULTADOS}

De los 365 pacientes evaluados en el estudio, 264 eran mujeres (72,3\%) y 101 eran varones. La edad media era de $81,1 \pm 6$ años, con extremos entre 65 y 96 años. Respecto al estado civil, $155(42,5 \%)$ eran casados, $23(6,3 \%)$ solteros, $186(51 \%)$ viudos y un paciente era divorciado $(0,3 \%)$. La mayoría de ellos vivía en su domicilio $(327 ; 89,6 \%)$, mientras que los 38 restantes $(10,4 \%)$ estaban institucionalizados. La media del número total de medicamentos fue de $5,5 \pm 2,5$, con 280 pacientes $(76,7 \%)$ que tomaban de forma crónica cuatro o más fármacos.

\section{Evaluación de la demencia}

Respecto al tipo de demencia, 289 pacientes $(79,1 \%)$ tenían EA, y los 76 $(21,9 \%)$ restantes, DV.

Había transcurrido una media de 28,5 \pm 28 meses desde el diagnóstico de la demencia. Al analizarse el estadio de la enfermedad según la escala GDS de Reisberg, existían 188 (51,5\%) pacientes con GDS3-5 y 177 (48,5\%) con GDS6-7.

Respecto al tratamiento específico para la demencia, 271 (74,2\%) pacientes tomaban algún tratamiento específico para la demencia, mientras que los 94 restantes $(25,8 \%)$ no tomaban ningún tratamiento específico en el momento de la evaluación. Recibían tratamiento con neurolépticos 200 (54,8\%) pacientes para el control de los síntomas conductuales y psicológicos.

\section{Valoración funcional}

Al evaluar las actividades instrumentales, la media del IL fue de $1,2 \pm 1,8$. Respecto a las actividades básicas de la vida diaria, la media del IB era 67,9 \pm 29 , con 60 pacientes $(16,4 \%)$ que aún mantenían un IB de 100 . Existían $142(38,9 \%)$ con un IB $<61$, y en $38(10,4 \%)$ pacientes el IB era $<21$. Al evaluar la cognición en el MMSE, se obtuvieron unas puntuaciones medias de $13,5 \pm 7$, con $103(28,2 \%)$ pacientes con valores del MMSE < 10 .

\section{Comorbilidad}

La media del índice de Charlson fue de 2,1 $\pm 1,3$. Respecto a los factores de riesgo cardiovascular, 197 pacientes (54\%) recibían tratamiento para la HTA, 97 pacientes $(26,6 \%)$ para la DM y 94 pacientes $(25,8 \%)$ para la DL. En 44 $(12,1 \%)$ pacientes existía el diagnóstico de insuficiencia cardíaca, en 50 $(13,7 \%)$ de EPOC, y en $39(10,6 \%)$ de cardiopatía isquémica. En $30(8,2 \%)$ casos coexistía el diagnóstico de neoplasia.

\section{Diferencias según el tipo de demencia}

En la tabla se muestran las principales diferencias encontradas al evaluar a los pacientes según el tipo de demencia. El análisis bivariable destaca diferencias en el género (mayor porcentaje de varones con DV), en la procedencia (predominio de pacientes institucionalizados en el grupo con DV), mejor puntuación del IB e IL en los pacientes con EA, más consumo de fármacos en los pacientes con DV y mayor posibilidad de recibir tratamiento específico para la demencia en los pacientes con EA. 
En los resultados del análisis multivariable persiste la significación con respecto a la DV y el ser varón $(p=0,02$; $\mathrm{OR}=2,43$; IC $95 \%=1,37-4,28)$, no recibir tratamiento específico para la demencia ( $p=0,0001 ; \mathrm{OR}=0,22 ;$ IC $95 \%$ $=0,13-0,39)$ y la toma de más de tres medicamentos $(p=$ $0,01 ; \mathrm{OR}=2,88 ;$ IC $95 \%=1,28-6,45)$.

\section{DISCUSIÓN}

El grado de comorbilidad de los pacientes con demencia varía según los diversos estudios [7-10,24]. Además, es probable que la comorbilidad en los pacientes con deterioro cognitivo frecuentemente esté infradiagnosticada y mal tratada [25]. Esto podría deberse a que los pacientes con demencia explican menos síntomas que los pacientes sin demencia en igualdad de comorbilidad [26].

En nuestro estudio, al explorar diferencias entre los dos tipos de demencia más frecuentes, tres variables persistieron significativas en el análisis multivariable. Así, existe un predominio de varones, un mayor porcentaje de pacientes con polifarmacia, así como un menor porcentaje de pacientes que reciben tratamiento con inhibidores de la acetilcolinesterasa y/o memantina en el grupo con DV.

En los estudios que evalúan diferencias de género según el tipo de demencia, los resultados muestran un predomino de EA en el género femenino y un predominio de DV en el varón [27]. Al evaluar las diferencias según el género en nuestro estudio, confirmamos la existencia de diferencias en el tipo de demencia según el género, de tal manera que las mujeres predominan en la EA y los varones en la DV [27,28].

Una manera indirecta que puede reflejar la comorbilidad es la cuantificación del número total de fármacos de prescripción crónica. En nuestro estudio, la media hallada de 5,5 fármacos es ligeramente inferior a la media de seis notificada en el grupo de 149 pacientes con demencia en el estudio de Lyketsos et al [4]; y ligeramente superior a la de 5,1 descrita por Schubert et al [8]. Hay que destacar el elevado porcentaje de pacientes que tomaba de forma crónica cuatro o más fármacos, especialmente en el grupo con DV.

Respecto a las diferencias en el tratamiento específico para la demencia en el sentido de que dicho tratamiento es menos frecuente prescrito en la DV, puede explicarse porque, a pesar de que existen algunos trabajos que demuestran la eficacia de estos fármacos en la DV [29-31], otros estudios muestran que tanto los inhibidores de la acetilcolinesterasa como la memantina producirían pequeños beneficios en la cognición, de significado clínico incierto, en pacientes con DV en grados leve-moderado [32]. Por ello, una recomendación es la de analizar de forma individualizada aquellos pacientes con DV que podrían beneficiarse de dicho tratamiento. Por otro lado, hay que tener en cuenta que el Consejo asesor para el tratamiento de la EA, organismo que regula en Cataluña la utilización de estos fármacos, no reconoce su indicación para la demencia vascular pura, y que ningún fármaco tiene la indicación en ficha técnica para DV, a
Tabla. Diferencias entre las variables evaluadas según el tipo de demencia.

\begin{tabular}{|c|c|c|c|}
\hline & $\begin{array}{l}\text { Alzheimer } \\
(n=289)\end{array}$ & $\begin{array}{l}\text { Demencia vascular } \\
\qquad(n=76)\end{array}$ & $p$ \\
\hline Género & & & 0,001 \\
\hline Masculino & $68(23,5 \%)$ & $33(43,4 \%)$ & \\
\hline Femenino & $221(76,5 \%)$ & $43(56,6 \%)$ & \\
\hline Edad (años) & $80,8 \pm 6,5$ & $81,6 \pm 6,4$ & 0,37 \\
\hline Estado civil & & & 0,7 \\
\hline Casados & $120(41,5 \%)$ & $35(46,1 \%)$ & \\
\hline Solteros & $17(5,9 \%)$ & $6(7,9 \%)$ & \\
\hline Viudos & $151(52,2 \%)$ & $35(46,1 \%)$ & \\
\hline Divorciados & $1(0,3 \%)$ & & \\
\hline Institucionalizados & $25(8,9 \%)$ & $13(17,1 \%)$ & 0,03 \\
\hline Índice de Barthel previo & $70,4 \pm 28$ & $58,3 \pm 29$ & 0,001 \\
\hline Índice de Lawton & $1,35 \pm 1,9$ & $0,84 \pm 1,4$ & 0,03 \\
\hline Test minimental & $14 \pm 6,6$ & $13,5 \pm 7,6$ & 0,56 \\
\hline Índice de Charlson & $2,11 \pm 1,3$ & $2 \pm 1,7$ & 0,87 \\
\hline Fármacos totales & $5,2 \pm 2,4$ & $6,5 \pm 2,6$ & 0,0001 \\
\hline Más de tres medicamentos & $212(73,4 \%)$ & $68(89,5 \%)$ & 0,003 \\
\hline Meses de diagnóstico previo & $29,8 \pm 26$ & $27,9 \pm 27$ & 0,73 \\
\hline Neoplasia & $26(9 \%)$ & $4(5,3 \%)$ & 0,29 \\
\hline Hipertensión arterial & $151(52,2 \%)$ & $46(60,5 \%)$ & 0,19 \\
\hline Diabetes mellitus & $79(27,3 \%)$ & $18(23,7 \%)$ & 0,71 \\
\hline Dislipemia & $74(25,6 \%)$ & $20(26,3 \%)$ & 0,67 \\
\hline $\begin{array}{l}\text { Enfermedad pulmonar } \\
\text { obstructiva crónica }\end{array}$ & $41(14,2 \%)$ & $9(11,8 \%)$ & 0,59 \\
\hline Insuficiencia cardíaca & $33(11,4 \%)$ & $11(14,5 \%)$ & 0,11 \\
\hline Cardiopatía isquémica & $32(11,1 \%)$ & $7(9,2 \%)$ & 0,64 \\
\hline $\begin{array}{l}\text { Tratamiento específico } \\
\text { de demencia }\end{array}$ & $233(80,6 \%)$ & $38(50 \%)$ & 0,0001 \\
\hline Tratamiento con neuroléptico & $162(56,1 \%)$ & $40(51,2 \%)$ & 0,45 \\
\hline Escala de deterioro global (GDS) & & & 0,06 \\
\hline $3-5$ & $156(53,9 \%)$ & $32(42,1 \%)$ & \\
\hline $6-7$ & $133(46,1 \%)$ & $44(57,9 \%)$ & \\
\hline
\end{tabular}

pesar de experiencias favorables con algunos fármacos, como muestra en una reciente revisión Robles-Bayón [33].

Entre las asociaciones que aparecieron en el análisis bivariable que perdieron su asociación en el multivariable, cabe destacar un mayor porcentaje de pacientes institucionalizados, así como un mayor deterioro en la realización de las actividades instrumentales y básicas de la vida diaria en los pacientes con DV. Nuestros resultados concuerdan con estudios previos en que, a igualdad de gravedad [34] o tiempo transcurrido [35] desde el diagnóstico de la demencia, los pacientes con EA resultan más independientes que los pacientes con DV. 
Muchos de los factores de riesgo para la DV, incluyendo HTA y DM, también incrementan el riesgo de EA [3]. En nuestro estudio no hallamos diferencias entre los dos tipos de demencia con los diversos factores de riesgo cardiovascular evaluados.

Respecto a algunas de las enfermedades crónicas más prevalentes (EPOC, insuficiencia cardíaca, etc.), los porcentajes son muy similares. Probablemente estos porcentajes con diagnósticos clínicos variarían si se dispusiera de estudios necrópsicos. En un interesante estudio de 52 pacientes diagnosticados de demencia de todos los tipos, y en que se disponía de autopsias, se comprobó que en el $20 \%$ de los pacientes existía evidencia de cardiopatía isquémica, y en el $73 \%$, de enfermedad ateroesclerótica cardiovascular [6].

Se han descrito diferencias respecto al perfil neuropsicológico de ambos tipos de demencia, con un patrón de alteración predominantemente 'cortical' en la demencia tipo EA y un patrón de afectación más subcortical en la DV [36]. En nuestro estudio no evaluamos los aspectos de la valoración neuropsicológica.

Entre las limitaciones del estudio, destaca que no se evaluó la intención de tratamiento, tampoco se recogieron los motivos para no iniciar tratamiento específico en los pacientes que no lo tomaban, ni los motivos de retirada en aquéllos en que se había suspendido una vez iniciado. También hay que considerar como limitación la posible existencia de un sesgo en la selección de la muestra, debido a la inclusión desde servicios de medicina interna y geriatría, pero no de neurología. Otro posible sesgo en la selección de la muestra radica en que existe un menor número de pacientes en el grupo de DV. Finalmente, hay que considerar que la recogida de antecedentes de factores de riesgo cardiovascular realizada puede dar algún error de clasificación, especialmente de infradiagnóstico.

En definitiva, ambos tipos de demencia (EA y DV) suelen acompañarse de importante comorbilidad médica en las personas mayores. Del mismo modo que el tener demencia complica el diagnóstico y manejo de otras enfermedades médicas, la comorbilidad puede convertir el tratamiento de la demencia en problemático. En este estudio podemos concluir que existen algunas variaciones en los pacientes mayores con demencia, dependiendo de si se trata de una EA o DV, destacando una mayor polifarmacia en los pacientes con DV.

\section{BIBLIOGRAFÍA}

1. Vilalta-Franch J, López-Pousa S, Llinàs-Reglà J. Prevalencia de demencias en una zona rural. Estudio Girona. Rev Neurol 2000; 30: 1026-32.

2. García FJ, Sánchez MI, Pérez A, Martín E, Marsal C, Rodríguez G, et al. Prevalencia de demencia y de sus subtipos principales en sujetos mayores de 65 años: efecto de la educación y ocupación. Estudio Toledo. Med Clin (Barc) 2001; 116: 401-7.

3. Kalaria R. Similarities between Alzheimer's disease and vascular dementia. Neurol Sci 2002; 203-204: 29-34.

4. Lyketsos CG, Toone L, Tschanz J, Rabins PV, Steinberg M, Onyike $\mathrm{CH}$, et al. Population-based study of medical comorbidity in early dementia and 'cognitive impairment, no dementia (CIND)': association with functional and cognitive impairment: the Cache County Study. Am J Geriatr Psychiatry 2005; 13: 656-64.

5. Doraiswamy PM, Leon J, Cummings JL, Marin D, Neumann PJ. Prevalence and impact of medical comorbidity in Alzheimer's disease. J Gerontol Med Sci 2002; 87: M173-7.

6. Fu C, Chute DJ, Farga ES, Garakian J, Cummings JF, Vinters HV. Comorbidity in dementia: an autopsy study. Arch Pathol Lab Med 2004; 128: 32-8.

7. Sanderson M, Wang J, Davis DR, Lane MJ, Cornman CB, Fadden MK. Co-morbidity associated with dementia. Am J Alzheimer Dis Other Demen 2002; 17: 73-8.

8. Schubert CC, Boustani M, Callahan CM, Perkins AJ, Carney CP, Fox $\mathrm{C}$, et al. Comorbidity profile of dementia patients in primary care: are they sicker? J Am Geriatr Soc 2006; 54: 104-9.

9. Zhu CW, Scarmeas N, Torgan R, Albert M, Brandt J, Blacker D, et al. Clinical features associated with costs in early AD. Neurology 2006; 66: 1021-8.

10. Artaz MA, Boddaert J, Hériche-Taillandier E, Dieudonné B, Verny M, le group REAL.FR. Medical comorbidity in Alzheimer's disease: baseline characteristics of the REAL.FR Cohort. Rev Med Interne 2006; 27: 91-7.

11. Albert SM, Costa R, Merchant C, Small S, Jenders RA, Stern Y. Hospitalization and Alzheimer's disease: results from a community-based study. J Gerontol A Biol Sci Med Sci 1999; 54: M267-71.

12. Van Dijk PT, Dippel DW, Van Der Meulen JH, Habbema JD. Comorbidity and its effect on mortality in nursing home patients with dementia. J Nerv Ment Dis 1996; 184: 180-7.

13. Formiga F, Fort I, Robles MJ, Barranco E, Espinosa MC, Riu S. Comorbilidad en pacientes ancianos con demencia. Diferencias por edad y género. Rev Clin Esp 2007; 207: 495-500.

14. American Psychiatric Association. Diagnostic and statistical manual of mental disorders. 4 ed. Washington DC: APA; 1993.

15. Mc Khann C, Drachman D, Folstein M, Katzman R, Price D, Stadlan EM. Clinical diagnosis of Alzheimer's disease: report of the NINCDSADRDA Work Group under the auspices of Department of Health and Human Services Task Force on Alzheimer's Disease. Neurology 1984; 34: 939-44.
16. Roman GC, Tatemichi TK, Erkinjuntti T, Cummings JL, Masdeu JC, García JH, et al. Vascular dementia: diagnostic criteria for research studies. Report of the NINDS-AIREN International Workshop. Neurology 1993; 43: 250-60.

17. Reisberg B, Ferris SH, De Leon MJ, Crok T. The Global Deterioration Scale for assessment of primary degenerative dementia. Am J Psychiatry 1982; 139: 1136-9.

18. Reisberg B. Functional Assessment Staging (FAST). Psychopharmacol Bull 1988; 24: 653-9.

19. Mahoney FI, Barthel DW. Functional evaluation: the Barthel index. A simple index of independence useful in scoring improvement in the rehabilitation of the chronically ill. Md State Med J 1965; 14: 61-5.

20. Cid-Rufaza J, Damián-Moreno J. Valoración de la discapacidad física: el índice de Barthel. Rev Esp Salud Publica 1997; 71: 127-37.

21. Lawton MP, Brody EM. Assessment of older people: self-maintaining and instrumental activities of daily living. Gerontologist 1969; 9: 179-86.

22. Folstein MF, Folstein SE, McHugh PR. 'Minimental state'. A practical method for grading the cognitive state of patients for the clinician. J Psychiatr Res 1975; 12: 189-98.

23. Charlson ME, Pompei P, Ales KL, MacKenzie CR. A new method of classifying prognostic comorbidity in longitudinal studies: development and validation J Chronic Dis 1987; 40: 373-83.

24. Reig L, Formiga F, Robles MJ, Fort I, Espinosa MC, Rodríguez D. Fibrilación auricular en los pacientes ancianos con demencia. Rev Esp Geriatr Gerontol 2007; 42: 84.

25. Lyketsos CG, Sheppard JMES, Rabins PV. Dementia in elderly persons in the general hospital. Am J Psychiatry 2000; 157: 704-7.

26. McCormick WC, Kukull WA, Van Belle G, Bowen JD, Teri L, Larson EB. Symptom patterns and comorbidity in the early stages of Alzheimer's disease. J Am Geriatr Soc 1994; 42: 517-21.

27. Ruitenberg A, Ott A, Van Swieten JC, Hofman A, Breteler MM. Incidence of dementia: does gender make a difference? Neurobiol Aging 2001: 22: 575-80.

28. Gambassi G, Lapane KL, Landi F, Sgadari A, Mor V, Bernadie R. Gender differences in the relation between comorbidity and mortality of patients with Alzheimer's disease. Neurology 1999; 53: 508-16.

29. Erkinjunti T, Kurz A, Gauthier S, Bullock R, Lilienfeld S, Damaraju CV. Efficacy of galantamine in probable vascular dementia and Alzheimer's disease combined with cerebrovascular disease: a randomised trial. Lancet 2002; 359: 1283-90.

30. Roman G, Wilkinson D, Doody R, Black S, Salloway S, Schindler RJ. Donepezil in vascular dementia: combined analysis of two large scale clinical trials. Dementia Geriatr Cogn Disord 2005; 20: 338-44.

31. Wilcock G, Möbius HJ, Stöffler A, MMM500 Group. A double-blind, placebo-controlled multicentre study of memantine in mild to moderate vascular dementia (MMM500). Int Clin Psychopharmacol 2002; 17 : 297-305.

32. Kavirajan H, Schneider LS. Efficacy and adverse effects of cholin- 
esterase inhibitors and memantine in vascular dementia: a meta-analysis of randomised controlled trials. Lancet Neurol 2007; 6: 782-92.

33. Robles-Bayón A. Acción de la memantina sobre la alteración cognitiva del paciente con demencia: reflexiones tras dos años de experiencia en España. Rev Neurol 2006; 42: 288-96.

34. Shiau MY, Yu L, Yuan HS, Lin JH, Liu CK. Functional performance of Alzheimer's disease and vascular dementia in Southern Taiwan. Kaohsiung J Med Sci 2006; 22: 437-46.
35. Groves WC, Brandt J, Steinberg M, Warren A, Rosenblatt A, Baker A, et al. Vascular dementia and Alzheimer disease: is there a difference? A comparison of symptoms by disease duration. J Neuropsychiatry Clin Neurosci 2000; 12: 305-15.

36. Álvarez-Saúco M, Moltó-Jordà JM, Morera-Guitart J, Frutos-Alegría MT, Matías-Guiu J. Actualización en el diagnóstico de la demencia vascular. Rev Neurol 2005; 41: 484-92.

\section{FEATURES DIFFERENTIATING COMORBIDITY IN ELDERLY PATIENTS}

WITH ALZHEIMER-TYPE DEMENTIA OR WITH VASCULAR DEMENTIA

Summary. Introduction. Alzheimer's disease $(A D)$ and vascular dementia $(V D)$ are the two most common forms of dementia. In the elderly subjects with dementia, there is a significant comorbidity associated and often cause greater morbidity and mortality. Aim. To investigate some aspects of comorbidity in the patients with these two types of dementia in order to analyze possible differences. Patients and methods. A total of 365 patients $>64$ years old were prospectively evaluated. Of them 289 patients (79.1\%) had probable AD, and 76 patients probable VD. Data were collected on sociodemographic variables, Barthel index, Lawton index), Minimental State Examination, total number of drugs, history of high blood pressure (HBP), diabetes mellitus $(D M)$, dyslipidemia $(D L)$, heart failure $(H F)$, chronic obstructive pulmonary disease (COPD) and cancer. Results. The sample consisted of 264 women (72.3\%) and 101 men. Mean age was $81.1 \pm 6$ years. Patients were taken an average of $5.5 \pm 2.5$ drugs. $54 \%$ had HBP, $26.6 \%$ DM, $25.8 \% \mathrm{DL}, 12.1 \% \mathrm{HF}, 13.7 \%$ COPD and $8.2 \%$ cancer. When in multivariate analysis differences were analyzed according the type of dementia, we found a higher percentage of men, taken more than three drugs and lower percentage of taken specific dementia therapy in the VD group. Conclusions. Our results showed the presence of high comorbidity and chronic drugs prescription in elderly people with dementia. There are some differences according the type of dementia, highlighting a higher percentage of polypharmacy in patients with vascular dementia group. [REV NEUROL 2008; 46: 72-6]

Key words. Alzheimer's disease. Cognitive impairment. Comorbidity. Elderly. Polypharmacy. Vascular dementia. 